\title{
Best practices for noninvasive ventilation
}

\author{
Andrew D. Bersten MBBS MD
}

See related review article by Keenan and colleagues, page E195

$\mathrm{N}$ oninvasive ventilation, specifically noninvasive positive-pressure ventilation or continuous positive airway pressure delivered by mask, has now become standard care for the management of a number of specific causes of acute respiratory failure. Noninvasive ventilation is a supportive measure. In respiratory diseases, for example, it is an important adjunct to optimal medical care such as bronchodilator therapy, corticosteroids and controlled oxygen therapy for chronic obstructive pulmonary disease (COPD).

The potential benefits of noninvasive ventilation for an acute exacerbation of COPD or for acute cardiogenic pulmonary edema were recognized more than 75 years ago. ${ }^{1}$ However, concerns about efficacy and safety have been successfully addressed only in the last 20 years. ${ }^{2}$

Noninvasive ventilation is postulated to improve abnormal physiologic effects of various diseases by reducing the work of breathing, improving oxygenation and alveolar ventilation and thereby reducing arterial carbon dioxide tension. These effects in turn can reduce the need for endotracheal mechanical ventilation as well as the rates of its associated complications, such as nosocomial infection, upper airway dysfunction, and adverse effects of sedation and muscle relaxation. Consequently, important outcomes evaluating noninvasive ventilation include mortality, rate of endotracheal intubation, complications and hospital length of stay.

In this issue of $C M A J$, Keenan and colleagues present clinical practice guidelines on the use of noninvasive ventilation in acute care settings based on their comprehensive review of 146 randomized controlled trials. ${ }^{2}$ They used GRADE methodology (Grading of Recommendations Assessment, Development and Evaluation), ${ }^{3}$ the most current form of methodologic analysis, to formulate the guidelines. Their focus on randomized clinical trials does limit their scope, but it also strengthens their recommendations and suggestions, which makes this an important document that should help reduce variations in practice and improve outcomes.

Keenan and colleagues recommend the use of noninvasive positive-pressure ventilation in addition to usual care in patients who have a severe exacerbation of COPD; this recommendation is supported by an impressive reduction in hospital mortality (relative risk [RR] 0.52, 95\% confidence interval [CI] 0.36-0.76). For patients who have respiratory failure due to cardiogenic pulmonary edema in the absence of shock or acute coronary syndrome requiring urgent coronary revascularization, the recommendation to use either form of noninvasive ventilation is supported by significant reductions in treatment failure (noninvasive positive-pressure ventilation: RR 0.36 , 95\% CI $0.25-0.51$; continuous positive airway pressure by mask: RR $0.23,95 \%$ CI $0.17-0.32$ ).

The use of noninvasive positive-pressure ventilation is suggested for immunosuppressed patients who have acute respiratory failure. Its use is also suggested to facilitate early weaning from endotracheal mechanical ventilation in patients with COPD and to facilitate the transition to spontaneous breathing after planned extubation in patients at high risk of recurrent respiratory failure, but only in centres that have expertise in this type of therapy.

\section{Failure of noninvasive ventilation}

Patients in whom noninvasive ventilation fails tend to have worse outcomes, probably because of delayed definitive care. Therefore, continuous positive airway pressure by mask is not recommended for patients who have acute lung injury. The association with serious adverse events such as cardiac arrest, which appears to reflect delayed endotracheal intubation, is not balanced by a reduction in mortality or rate of

\section{- KEY POINTS}

- Successful implementation of programs for noninvasive ventilation requires 24-hour availability of an experienced team and appropriate selection of patients.

- Patients in whom noninvasive ventilation fails tend to have worse outcomes, probably because of delayed definitive care.

- Any program must include adequate monitoring of patients to ensure a rapid response when noninvasive ventilation fails.
Competing interests: Andrew Bersten received fees and travel assistance from ResMed Ltd. for a one-day consultation regarding noninvasive ventilation.

This article was solicited and has not been peer reviewed.

Correspondence to: Dr. Andrew D. Bersten, andrew.bersten@flinders .edu.au

CMAJ 2011. DOI:10.1503 /cmaj.110122 
endotracheal intubation. Similarly, in a multicentre observational study, Demoule and coauthors reported an association between failure of noninvasive ventilation, defined as subsequent endotracheal intubation or death before intubation, with an increased length of stay among patients with a severe exacerbation of COPD or acute pulmonary edema, and an increase in the rate of death from other causes of acute respiratory failure. ${ }^{4}$

Failure of noninvasive ventilation needs to be detected early, followed by rapid access to safe endotracheal intubation or palliation. In patients with exacerbation of COPD, failure of noninvasive ventilation is associated with failure to improve hypercapnic acidosis or respiratory rate in the first few hours of treatment, high acuity of illness and lack of patient cooperation. ${ }^{5}$ However, using metaregression analysis, Peter and Moran found that the log odds ratio of intubation is inversely related to the severity of hypercapnic acidosis. ${ }^{6}$ In other words, within the limited range of severity studied, patients with more severe hypercapnic acidosis are more likely to benefit from noninvasive positive-pressure ventilation, but the ventilation is also more likely to fail. Therefore, the use of noninvasive positivepressure ventilation is recommended for patients who have a severe exacerbation of COPD and an arterial $\mathrm{pH}$ of less than 7.35. It is not helpful for those with an arterial $\mathrm{pH}$ of 7.35 or higher because of the lack of evidence of benefit.

Early detection of failed noninvasive ventilation requires careful monitoring of the patient's vital signs and blood gas levels. Rapid access to conventional mechanical ventilation will depend on the local health care setting and where the noninvasive ventilation is being administered. In a multicentre randomized controlled trial, Plant and colleagues showed that noninvasive ventilation in patients with a severe exacerbation of COPD was safe and effective in the general ward setting. ${ }^{7}$ However, outcomes of patients in general wards who had more severe disease (arterial $\mathrm{pH}<7.30^{7}$ ) were not as good as those reported for patients in acute care locations, ${ }^{8}$ where more sophisticated equipment and individual titration of ventilator settings were available.

\section{Effective implementation}

Many factors appear to influence the effective implementation of noninvasive ventilation. These factors include an experienced team of health care staff able to provide 24-hour service and detailed attention to mask interface and leaks, choice of equipment, ventilator settings, inspired oxygen levels, glottic function and clearance of secretions. Failure of the ventilator to match changing patient demand can occur at the start, during and at the end of inspiration. This patient-ventilator dyssynchrony is common, often goes unrecognized and contributes to the failure of noninvasive ventilation. Appropriate monitoring is essential, along with staffing schedules that recognize the usually high nursing workload in the first six to eight hours.' The role of humidification and the optimal period of rest from noninvasive ventilation to allow oral intake and speech remain under investigation.

Given the increasing demand for critical care beds, not all patients receiving noninvasive ventilation may be cared for in intensive care units or emergency departments. Adequate monitoring is required wherever the patient is located. To facilitate the rescue of patients in whom noninvasive ventilation fails, and to maximize overall benefits of noninvasive ventilation programs, monitoring of patients is best done in partnership with the many services and specialties caring for acutely ill patients.

For these guidelines ${ }^{2}$ to change clinical practice, they have to be supported by appropriate education, implementation and review. Helping clinicians know when and when not to use noninvasive ventilation is perhaps the most important role for these guidelines.

\section{References}

1. Poulton PE. Left-sided heart failure with pulmonary oedema: its treatment with the "pulmonary plus pressure machine." Lancet 1936;2:981-3

2. Keenan S, Sinuff T, Burns K, et al. Clinical practice guidelines for the use of noninvasive positive-pressure ventilation and noninvasive continuous positive airway pressure in the acute care setting. CMAJ 2011;183:331-8.

3. GRADE Working Group. Grading quality of evidence and strength of recommendations. BMJ 2004;328:1-3.

4. Demoule A, Taille S, Richard JC, et al. Benefits and risks of success or failure of noninvasive ventilation. Intensive Care Med 2006;32:1756-65.

5. Nava S, Hill N. Noninvasive ventilation in acute respiratory failure. Lancet 2009;374:250-9.

6. Peter JV, Moran JL. Noninvasive ventilation in exacerbations of chronic obstructive pulmonary disease: implications of different meta-analytic strategies. Ann Intern Med 2004;141:W78-9.

7. Plant PK, Owen JL, Elliott MW. Early use of non-invasive ventilation for acute exacerbations of chronic obstructive pulmonary disease on general respiratory wards: a multicentre randomised controlled trial. Lancet 2000;355:1931-5.

8. Elliott MW, Confalonieri M, Nava S. Where to perform noninvasive ventilation? Eur Respir J 2002;19:1159-66.

9. Brochard L. Noninvasive ventilation for acute respiratory failure. JAMA 2002;288:932-5.

Affiliation: Dr. Andrew Bersten is Professor and Director of the Intensive and Critical Care Unit, Flinders Medical Centre, Adelaide, Australia. 